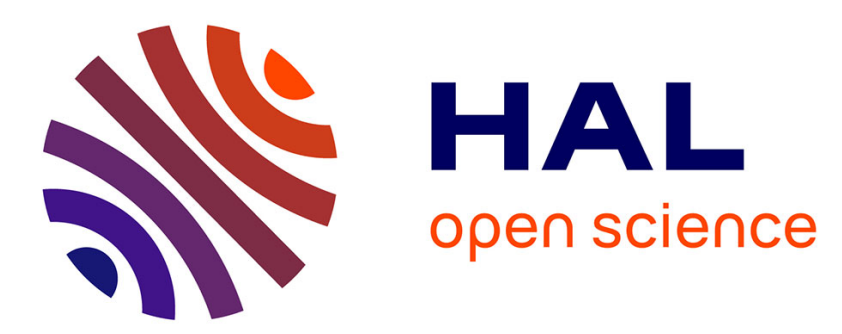

\title{
A new family of polyno-expo-trigonometric distributions with applications
}

Farrukh Jamal, Christophe Chesneau

\section{To cite this version:}

Farrukh Jamal, Christophe Chesneau. A new family of polyno-expo-trigonometric distributions with applications. 2019. hal-02049768v2

\section{HAL Id: hal-02049768 \\ https://hal.science/hal-02049768v2}

Preprint submitted on 12 May 2019

HAL is a multi-disciplinary open access archive for the deposit and dissemination of scientific research documents, whether they are published or not. The documents may come from teaching and research institutions in France or abroad, or from public or private research centers.
L'archive ouverte pluridisciplinaire HAL, est destinée au dépôt et à la diffusion de documents scientifiques de niveau recherche, publiés ou non, émanant des établissements d'enseignement et de recherche français ou étrangers, des laboratoires publics ou privés. 


\title{
A NEW FAMILY OF POLYNO-EXPO-TRIGONOMETRIC DISTRIBUTIONS WITH APPLICATIONS
}

\author{
FARRUKH JAMAL AND CHRISTOPHE CHESNEAU
}

\begin{abstract}
In this paper, a new family of polyno-expo-trigonometric distributions is presented and investigated. A special case using the Weibull distribution, with three parameters, is considered as statistical model for lifetime data. The estimation of the parameters are performed with the maximum likelihood method. A numerical simulation study verifies that the empirical bias and mean squared error of the obtained maximum likelihood estimates tend to zero as the sample size increases. Three real life data sets are then analyzed. We show that our model has a good fit in comparison to other well-known powerful models in the literature.
\end{abstract}

Keywords: Trigonometric distribution, Probability density function, Weibull distribution, Maximum likelihood estimation.

$M S C 2010$ 60E 05, 62E 15.

\section{INTRODUCTION}

Over the last decades, the modeling and prediction of physical phenomena have been improved considerably thanks to the emergence of various families of probability distributions. The construction of statistical models which capture possible small and/or complex variations in such phenomena still remains a great challenge for the statistician. To reach this goal, nonstandard distributions involving trigonometric functions in the definition of the probability density function (pdf) have been developed. Among the most useful of them, let us mention the sine distribution (see [11]), the cosine distribution (see [15]), the beta trigonometric distribution (see [14]), the Von Mises distribution (see [9] and [17]), the sine square distribution (see [2]), the sin-skew logistic distribution (see [6]), the SS distribution (see [13]) and the CS distribution (see [7]). All these references show that trigonometric functions can play an important role in statistical analysis with great impacts in various applications. They motivate deeper research in this direction. This paper provides a contribution to the subject. Based on a new theoretical result, we introduce and study a new family of distributions involving trigonometric functions. To be more specific, the related pdf has the feature to be defined by a quotient of two functions using polynomial, exponential, sine and cosine functions. The richness of this definition have the merit to ensure a great flexibility of the pdf, opening the door to the construction of 
statistical models with goodness of fit. A special focus is done on a new distribution with three parameters, using the Weibull distribution as baseline distribution. We show in what contact it can be used as a statistical model for lifetime data.

The remaining part of this paper is organized as follows. Our new family of distributions is introduced in Section 2, with some interesting special cases, including the one using the Weibull distribution. In Section 3, maximum likelihood estimates of the parameters of interest are investigated and simulation results assess their convergence. The importance of our family of distributions is illustrated in Section 4 by considering three real life data sets. The proofs of our theoretical result is postponed in Section 5 .

\section{A NEW FAMily OF Distributions}

2.1. A theoretical result. The result below presents a new cumulative distribution function (cdf) of a random variable with support $\left[0, \frac{\pi}{2}\right]$ using polynomial, exponential and trigonometric functions.

Theorem 2.1. For any $a \geq 0, b \in[0,1]$ and $c \geq 0$, let $A(y)$ be the function defined by

$$
A(y)=\left(c+\frac{\pi}{2} e^{-a \frac{\pi}{2}}\right) \frac{\sin (y)-b y e^{-a y} \cos (y)}{\cos (y)+\left(c+y e^{-a y}\right) \sin (y)}, \quad y \in\left[0, \frac{\pi}{2}\right] .
$$

Then, the function $B(y)$ defined below possesses the properties of a cdf:

$$
B(y)= \begin{cases}1 & \text { if } y>\frac{\pi}{2}, \\ A(y) & \text { if } y \in\left[0, \frac{\pi}{2}\right], \\ 0 & \text { if } y<0 .\end{cases}
$$

The proof of this result is postponed in Section 5. To the best of our knowledge, the cdf $B(y)$ given by (1) is new in the literature. Following the spirit of complex trigonometric distributions (see [13] and [7] for instance), let us mention that it is based on a mathematical definition only; its is not based on stochastic representation or physical interpretation.

2.2. Genesis of the family. Starting from the new cdf $B(y)$ given by (1), we are able to create a wide variety of distributions from a former cdf $F(x)$. More precisely, let $F(x)$ be a cdf and $G(x)$ be the function defined by $G(x)=B\left(\frac{\pi}{2} F(x)\right)=$ $A\left(\frac{\pi}{2} F(x)\right)$, i.e.

$$
\begin{aligned}
G(x)= & \left(c+\frac{\pi}{2} e^{-a \frac{\pi}{2}}\right) \frac{\sin \left(\frac{\pi}{2} F(x)\right)-b \frac{\pi}{2} F(x) e^{-a \frac{\pi}{2} F(x)} \cos \left(\frac{\pi}{2} F(x)\right)}{\cos \left(\frac{\pi}{2} F(x)\right)+\left(c+\frac{\pi}{2} F(x) e^{-a \frac{\pi}{2} F(x)}\right) \sin \left(\frac{\pi}{2} F(x)\right)}, \\
& x \in \mathbb{R} .
\end{aligned}
$$


Then, $G(x)$ is a cdf. Indeed, since $B(y)$ has the properties of a cdf of a random variable with support $\left[0, \frac{\pi}{2}\right]$ by Theorem 2.1 and $\frac{\pi}{2} F(x) \in\left[0, \frac{\pi}{2}\right]$, then $G(x)$ has the properties of a cdf by composition with respect to the support. For the sake of conciseness, the family of distributions characterized by $G(x)$ will be denoted by TransSC $(F ; a, b, c)$ (as Transformation of distributions using Sine and Cosine functions). In the same spirit, contrary to those in [13] or in [7] defined as a quotient of trigonometric functions only, it has the advantage to modulate functions of various natures: polynomial, exponential and trigonometric. This point is crucial, as we shall see in the application section (Section 4). Moreover, observe that the parameter $a$ is related to the exponential term, $b$ is related to the cosine term in the numerator and $c$ has influence in the denominator as a sole variable.

Remark 1. For $x$ such that $F(x) \neq 1$, an alternative expression of $G(x)$ in term of tangent function is given by

$$
G(x)=\left(c+\frac{\pi}{2} e^{-a \frac{\pi}{2}}\right) \frac{\tan \left(\frac{\pi}{2} F(x)\right)-b \frac{\pi}{2} F(x) e^{-a \frac{\pi}{2} F(x)}}{1+\left(c+\frac{\pi}{2} F(x) e^{-a \frac{\pi}{2} F(x)}\right) \tan \left(\frac{\pi}{2} F(x)\right)}, \quad x \in \mathbb{R} .
$$

As usual, the pdf of the TransSC $(F ; a, b, c)$ distribution is defined by $g(x)=G^{\prime}(x)$ almost surely. Due to the massiveness of its expression we omit it here; it is however given in the proof of Theorem 2.1. Some special cases of the $\operatorname{TransSC}(F ; a, b, c)$ distribution with a more tractable pdfs are presented below.

2.3. Some special cases. First of all, we consider the $\operatorname{TransSC}(F ; 0,0, c)$ distribution, corresponding to $\operatorname{TransSC}(F ; a, b, c)$ with $a=0, b=0$ and $c \geq 0$. The cdf given by (3) becomes

$$
G(x)=\left(c+\frac{\pi}{2}\right) \frac{\sin \left(\frac{\pi}{2} F(x)\right)}{\cos \left(\frac{\pi}{2} F(x)\right)+\left(c+\frac{\pi}{2} F(x)\right) \sin \left(\frac{\pi}{2} F(x)\right)}, \quad x \in \mathbb{R} .
$$

The pdf is given by

$$
g(x)=\frac{\pi}{2}\left(c+\frac{\pi}{2}\right) \frac{\cos ^{2}\left(\frac{\pi}{2} F(x)\right) f(x)}{\left[\cos \left(\frac{\pi}{2} F(x)\right)+\left(c+\frac{\pi}{2} F(x)\right) \sin \left(\frac{\pi}{2} F(x)\right)\right]^{2}} .
$$

The fact that $G(x)$ has the properties of a cdf is immediate. Table 1 presents some special cases of the $\operatorname{TransSC}(F ; 0,0, c)$ distribution with well-known cdfs $F(x)$ of random variables of various support.

Let us now consider the $\operatorname{TransSC}(F ; 0, b, 0)$ distribution, corresponding to $\operatorname{TransSC}(F ; a, b, c)$ with $a=0, b \in[0,1]$ and $c=0$. The cdf given by (3) becomes

$$
G(x)=\frac{\pi}{2} \frac{\sin \left(\frac{\pi}{2} F(x)\right)-b \frac{\pi}{2} F(x) \cos \left(\frac{\pi}{2} F(x)\right)}{\cos \left(\frac{\pi}{2} F(x)\right)+\frac{\pi}{2} F(x) \sin \left(\frac{\pi}{2} F(x)\right)}, \quad x \in \mathbb{R} .
$$


TABle 1. Some special cases of the $\operatorname{TransSC}(F ; 0,0, c)$ distribution with the related pdf $g(x)$.

\begin{tabular}{|c|c|c|c|}
\hline$F(x)$ & Support & $g(x)$ & Param. \\
\hline Uniform & $(0, \alpha)$ & $\frac{\pi}{2 \alpha}\left(c+\frac{\pi}{2}\right) \frac{\cos ^{2}\left(\frac{\pi}{2 \alpha} x\right)}{\left[\cos \left(\frac{\pi}{2 \alpha} x\right)+\left(c+\frac{\pi}{2 \alpha} x\right) \sin \left(\frac{\pi}{2 \alpha} x\right)\right]^{2}}$ & $(\alpha, c)$ \\
\hline Exp. & $(0,+\infty)$ & $\frac{\pi}{2}\left(c+\frac{\pi}{2}\right) \frac{\sin ^{2}\left(\frac{\pi}{2} e^{-\alpha x}\right) \alpha e^{-\alpha x}}{\left[\sin \left(\frac{\pi}{2} e^{-\alpha x}\right)+\left(c+\frac{\pi}{2}\left(1-e^{-\alpha x}\right)\right) \cos \left(\frac{\pi}{2} e^{-\alpha x}\right)\right]^{2}}$ & $(\alpha, c)$ \\
\hline Weibull & $(0,+\infty)$ & $\frac{\pi}{2}\left(c+\frac{\pi}{2}\right) \frac{\sin ^{2}\left(\frac{\pi}{2} e^{-\alpha x}\right) \alpha \beta x^{\beta-1} e^{-\alpha x^{2}}}{\left[\sin \left(\frac{\pi}{2} e^{-\alpha x^{\beta}}\right)+\left(c+\frac{\pi}{2}\left(1-e^{-\alpha x^{\beta}}\right)\right) \cos \left(\frac{\pi}{2} e^{-\alpha x^{\beta}}\right)\right]^{2}}$ & $(\alpha, \beta, c$ \\
\hline Fréchet & $(0,+\infty)$ & 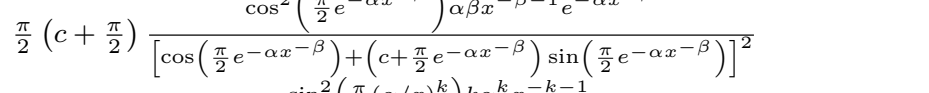 & $(\alpha, \beta, c$ \\
\hline Pareto & $(\alpha,+\infty)$ & $\frac{\pi}{2}\left(c+\frac{\pi}{2}\right) \frac{\sin ^{2}\left(\frac{\pi}{2}(\alpha / x)^{k}\right) k \alpha^{k} x^{-k-1}}{\left[\sin \left(\frac{\pi}{2}(\alpha / x)^{k}\right)+\left(c+\frac{\pi}{2}\left(1-(\alpha / x)^{k}\right)\right) \cos \left(\frac{\pi}{2}(\alpha / x)^{k}\right)\right]^{2}}$ & $(\alpha, k, c)$ \\
\hline Lomax & $(0,+\infty)$ & $\frac{\pi}{2}\left(c+\frac{\pi}{2}\right) \frac{\sin ^{2}\left(\frac{\pi}{2}(1+(x / s))^{-k}\right)(k / s)(1+(x / s))^{-k-1}}{\left[\sin \left(\frac{\pi}{2}(1+(x / s))^{-k}\right)+\left(c+\frac{\pi}{2}\left(1-(1+(x / s))^{-k}\right)\right) \cos \left(\frac{\pi}{2}(1+(x / s))^{-k}\right)\right]^{2}}$ & $(s, k, c)$ \\
\hline Normal & $\mathbb{R}$ & $\frac{\pi}{2}\left(c+\frac{\pi}{2}\right) \frac{\cos ^{2}\left(\frac{\pi}{2} \Phi((x-\mu) / \sigma)\right)\left(1 / \sqrt{2 \pi \sigma^{2}}\right) \mathrm{e}^{-(x-\mu)^{2} / \sigma^{2}}}{\left[\cos \left(\frac{\pi}{2} \Phi((x-\mu) / \sigma)\right)+\left(c+\frac{\pi}{2} \Phi((x-\mu) / \sigma)\right) \sin \left(\frac{\pi}{2} \Phi((x-\mu) / \sigma)\right)\right]^{2}}$ & $(\mu, \sigma, c)$ \\
\hline
\end{tabular}

The pdf is given by

$$
g(x)=\frac{\pi^{2}}{2^{4}} \frac{\left[b \pi^{2}(F(x))^{2}+4(1-b) \cos ^{2}\left(\frac{\pi}{2} F(x)\right)\right] f(x)}{\left[\cos \left(\frac{\pi}{2} F(x)\right)+\frac{\pi}{2} F(x) \sin \left(\frac{\pi}{2} F(x)\right)\right]^{2}}, \quad x \in \mathbb{R} .
$$

Again, note that the fact that $G(x)$ has the properties of a cdf is immediate.

To illustrate the crucial role of $b$ in the nature of $g(x)$, let us remark that, when $b=0$, it is reduced to

$$
g(x)=g_{0}(x)=\frac{\pi^{2}}{4} \frac{\cos ^{2}\left(\frac{\pi}{2} F(x)\right) f(x)}{\left[\cos \left(\frac{\pi}{2} F(x)\right)+\frac{\pi}{2} F(x) \sin \left(\frac{\pi}{2} F(x)\right)\right]^{2}}, \quad x \in \mathbb{R} .
$$

When $b=1$, the trigonometric term in the numerator disappears; $g(x)$ is reduced to

$$
g(x)=g_{1}(x)=\frac{\pi^{4}}{2^{4}} \frac{(F(x))^{2} f(x)}{\left[\cos \left(\frac{\pi}{2} F(x)\right)+\frac{\pi}{2} F(x) \sin \left(\frac{\pi}{2} F(x)\right)\right]^{2}}, \quad x \in \mathbb{R} .
$$

Remark 2. Another point of view is that $g(x)$ is a mixture of 2 pdfs: $g_{1}(x)$ and $g_{0}(x)$ with weights $(b, 1-b)$. Indeed, we can write $g(x)=b g_{1}(x)+(1-b) g_{0}(x)$.

Table 2 shows some special cases of the $\operatorname{TransSC}(F ; 0, b, 0)$ distribution with well-known cdfs $F(x)$ of various support.

We can now investigate the $\operatorname{TransSC}(F ; a, 0,0)$ distribution, corresponding to $\operatorname{TransSC}(F ; a, b, c)$ with $a \geq 0, b=0$ and $c=0$. The cdf given by (3) becomes

$$
G(x)=\frac{\pi}{2} e^{-a \frac{\pi}{2}} \frac{\sin \left(\frac{\pi}{2} F(x)\right)}{\cos \left(\frac{\pi}{2} F(x)\right)+\frac{\pi}{2} F(x) e^{-a \frac{\pi}{2} F(x)} \sin \left(\frac{\pi}{2} F(x)\right)}, \quad x \in \mathbb{R} .
$$


TABle 2. Some special cases of the $\operatorname{TransSC}(F ; 0, b, 0)$ distribution with the related pdf $g(x)$.

\begin{tabular}{|c|c|c|c|}
\hline$F(x)$ & Support & $g(x)$ & Param. \\
\hline Uniform & $(0, \alpha)$ & $\frac{\pi^{2}}{2^{4} \alpha} \frac{\left[b \pi^{2}\left(\frac{x}{\alpha}\right)^{2}+4(1-b) \cos ^{2}\left(\frac{\pi}{2 \alpha} x\right)\right]}{\left[\cos \left(\frac{\pi}{2} x\right)+\frac{\pi}{\pi} x \sin \left(\frac{\pi}{\pi} x\right)\right]^{2}}$ & $(\alpha, b)$ \\
\hline Exp. & $(0,+\infty)$ & 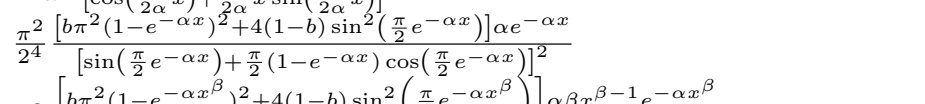 & $(\alpha, b)$ \\
\hline Weibull & $(0,+\infty)$ & $\begin{array}{c}\frac{\pi^{2}}{2^{4}} \frac{\left[b \pi^{2}\left(1-e^{-\alpha x^{\beta}}\right)^{2}+4(1-b) \sin ^{2}\left(\frac{\pi}{2} e^{-\alpha x^{\beta}}\right)\right] \alpha \beta x^{\beta-1} e^{-\alpha x^{\beta}}}{\left[\sin \left(\frac{\pi}{2} e^{-\alpha x^{\beta}}\right)+\frac{\pi}{2}\left(1-e^{-\alpha x^{\beta}}\right) \cos \left(\frac{\pi}{2} e^{-\alpha x^{\beta}}\right)\right]^{2}} \\
{\left[b \pi^{2} e^{-2 \alpha x^{-\beta}}+4(1-b) \cos ^{2}\left(\frac{\pi}{2} e^{-\alpha x}-\beta\right)\right]_{\alpha \beta x^{-\beta-1} e^{-\alpha x^{-\beta}}}}\end{array}$ & $(\alpha, \beta, b)$ \\
\hline Fréchet & $(0,+\infty)$ & $\frac{\pi^{2}}{2^{4}} \frac{\left.\left[{ }^{6 \pi^{2} e^{-\alpha x}}+4(1-b) \cos ^{2}\left(\frac{\pi}{2} e^{-\alpha x}\right)\right]\right]^{\alpha \beta x}}{\left[\cos \left(\frac{\pi}{2} e^{-\alpha x^{-\beta}}\right)+\frac{\pi}{2} e^{-\alpha x^{-\beta}} \sin \left(\frac{\pi}{2} e^{-\alpha x^{-\beta}}\right)\right]^{2}}$ & $(\alpha, \beta, b)$ \\
\hline Pareto & $(\alpha,+\infty)$ & $\frac{\pi^{2}}{2^{4}} \frac{\left[b \pi^{2}\left(1-(\alpha / x)^{k}\right)^{2}+4(1-b) \sin ^{2}\left(\frac{\pi}{2}(\alpha / x)^{k}\right)\right] k \alpha^{k} x^{-k-1}}{\left[\sin \left(\frac{\pi}{2}(\alpha / x)^{k}\right)+\frac{\pi}{2}\left(1-(\alpha / x)^{k}\right) \cos \left(\frac{\pi}{2}(\alpha / x)^{k}\right)\right]^{2}}$ & $(\alpha, k, b)$ \\
\hline Lomax & $(0,+\infty)$ & $\frac{\pi^{2}}{2^{4}} \frac{\left[b \pi^{2}\left(1-(1+(x / s))^{-k}\right)^{2}+4(1-b) \sin ^{2}\left(\frac{\pi}{2}(1+(x / s))^{-k}\right)\right](k / s)(1+(x / s))^{-k-1}}{\left[\sin \left(\frac{\pi}{2}(1+(x / s))^{-k}\right)+\frac{\pi}{2}\left(1-(1+(x / s))^{-k}\right) \cos \left(\frac{\pi}{2}(1+(x / s))^{-k}\right)\right]^{2}}$ & $(s, k, b)$ \\
\hline Normal & $\mathbb{R}$ & $\frac{\pi^{2}}{2^{4}} \frac{\left[b \pi^{2}(\Phi((x-\mu) / \sigma))^{2}+4(1-b) \cos ^{2}\left(\frac{\pi}{2} \Phi((x-\mu) / \sigma)\right)\right]\left(1 / \sqrt{2 \pi \sigma^{2}}\right) \mathrm{e}^{-(x-\mu)^{2} / \sigma^{2}}}{\left[\cos \left(\frac{\pi}{2} \Phi((x-\mu) / \sigma)\right)+\frac{\pi}{2} \Phi((x-\mu) / \sigma) \sin \left(\frac{\pi}{2} \Phi((x-\mu) / \sigma)\right)\right]^{2}}$ & $(\mu, \sigma, b)$ \\
\hline
\end{tabular}

The pdf is given by

$$
g(x)=\frac{\pi^{2}}{2^{3}} e^{-a \frac{\pi}{2}(1+F(x))} \frac{\left[2 e^{a \frac{\pi}{2} F(x)}-(2-a \pi F(x)) \sin ^{2}\left(\frac{\pi}{2} F(x)\right)\right] f(x)}{\left[\cos \left(\frac{\pi}{2} F(x)\right)+\frac{\pi}{2} F(x) e^{-a \frac{\pi}{2} F(x)} \sin \left(\frac{\pi}{2} F(x)\right)\right]^{2}}, \quad x \in \mathbb{R}
$$

Standard mathematical arguments prove that $G(x)$ has the properties of a cdf.

2.4. The $\operatorname{TSCW}(\alpha, \beta, b)$ distribution. Among all the special cases of the $\operatorname{TransSC}(F$; $a, b, c)$ distribution presented in the previous subsection, thanks to its faculty to possibly vanish the trigonometric term in the numerator of its pdf, we focus our attention on the TransSC $(F ; 0, b, 0)$ distribution. Moreover, in order to construct a lifetime distribution with a great flexibility in the pdf, we consider the baseline cdf $F(x)$ of the well-known Weibull distribution: $F(x)=1-e^{-\alpha x^{\beta}}, \alpha, \beta, x>0$. The related distribution is denoted by $\operatorname{TSCW}(\alpha, \beta, b)$ or $\operatorname{TSCW}$ for short. Then, the pdf (4) becomes

$$
g(x)=\frac{\pi^{2}}{2^{4}} \frac{\left[b \pi^{2}\left(1-e^{-\alpha x^{\beta}}\right)^{2}+4(1-b) \sin ^{2}\left(\frac{\pi}{2} e^{-\alpha x^{\beta}}\right)\right] \alpha \beta x^{\beta-1} e^{-\alpha x^{\beta}}}{\left[\sin \left(\frac{\pi}{2} e^{-\alpha x^{\beta}}\right)+\frac{\pi}{2}\left(1-e^{-\alpha x^{\beta}}\right) \cos \left(\frac{\pi}{2} e^{-\alpha x^{\beta}}\right)\right]^{2}}, \quad x>0
$$


The hazard rate function (hrf) is given by

$$
\begin{aligned}
h(x) & =\frac{g(x)}{1-G(x)} \\
& =\frac{\pi^{2}}{2^{4}} \frac{\left[b \pi^{2}\left(1-e^{-\alpha x^{\beta}}\right)^{2}+4(1-b) \sin ^{2}\left(\frac{\pi}{2} e^{-\alpha x^{\beta}}\right)\right] \alpha \beta x^{\beta-1} e^{-\alpha x^{\beta}}}{\left[\sin \left(\frac{\pi}{2} e^{-\alpha x^{\beta}}\right)+\frac{\pi}{2}\left(1-e^{-\alpha x^{\beta}}\right) \cos \left(\frac{\pi}{2} e^{-\alpha x^{\beta}}\right)\right]} \times \\
& \frac{1}{\left[\sin \left(\frac{\pi}{2} e^{-\alpha x^{\beta}}\right)-\frac{\pi}{2} \cos \left(\frac{\pi}{2} e^{-\alpha x^{\beta}}\right) e^{-\alpha x^{\beta}}+b\left(\frac{\pi}{2}\right)^{2}\left(1-e^{-\alpha x^{\beta}}\right) \sin \left(\frac{\pi}{2} e^{-\alpha x^{\beta}}\right)\right]}, \\
& x>0 .
\end{aligned}
$$

Some plots of the pdf (5) and hrf (6) are displayed in Figures 1 and 2 for selected parameters values for $(\alpha, \beta, b)$. The flexibility of the curves shapes can be observed, illustrating the versatility of the $\operatorname{TSCW}(\alpha, \beta, b)$ distribution.
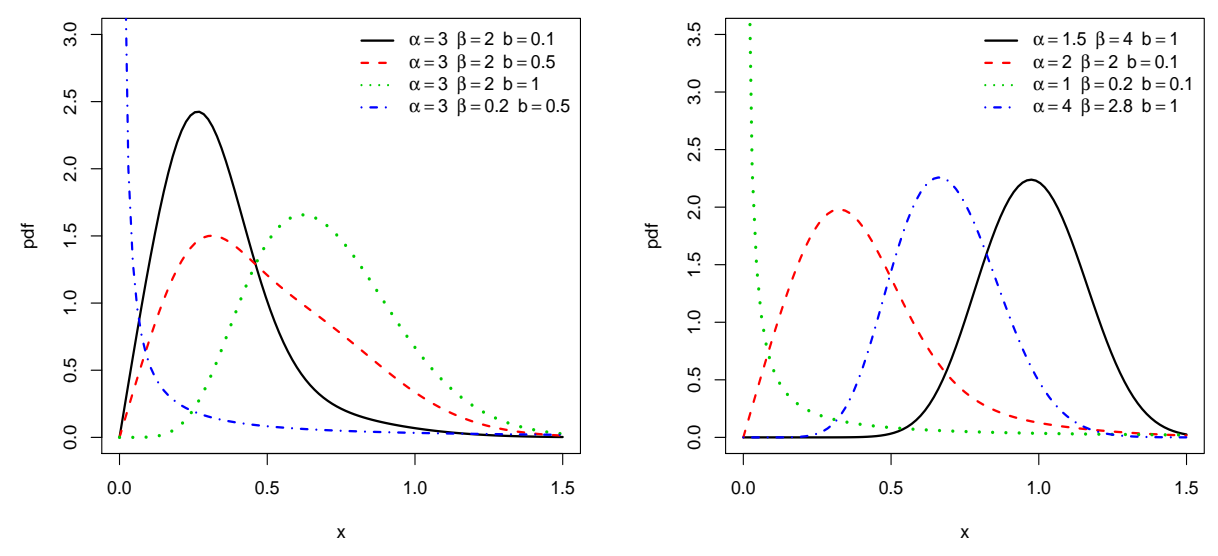

Figure 1. Plots for pdf of the TSCW distribution.

In the rest of this study, we focus our attention on the $\operatorname{TCSW}(\alpha, \beta, b)$ distribution and prove its merits in terms of modelling in prediction.

\section{Simulation Study}

In this section we provide a graphical Monte Carlo simulation study which aims to compare the precision of the different maximum likelihood estimates (MLEs) of the supposed unknown parameters $(\alpha, \beta, b)$ of the TCSW distribution. The MLEs of $\alpha, \beta$ and $b$ are naturally denoted by $\hat{\alpha}, \hat{\beta}$ and $\hat{b}$ respectively. The computations are performed by using $\mathrm{R}$ programs. We generate $N=1000$ samples of different 

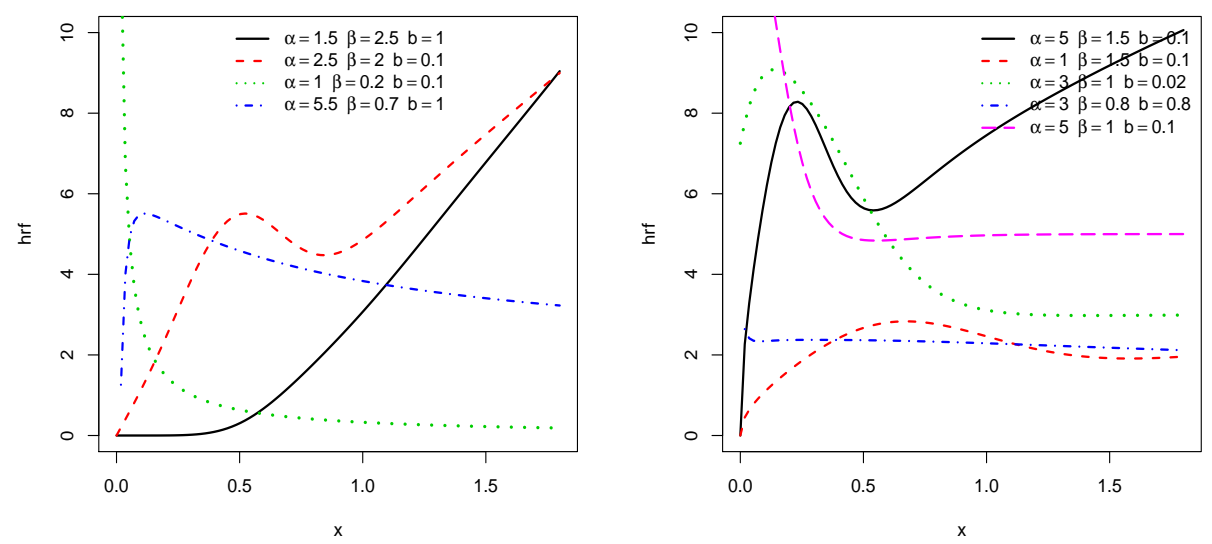

Figure 2. Plots for hrf of the TSCW distribution.

sizes: $n=20,25, \ldots, 300$ from TCSW distribution with the given parameters values $\alpha=2, \beta=1.5$ and $b=0.3$. We also calculate the empirical bias and mean square error (MSE) of the MLEs. For $h=\alpha, \beta$ and $b$ in turn, they are respectively defined by

$$
\operatorname{Bias}_{h}=\frac{1}{N} \sum_{i=1}^{N}\left(\hat{h}_{i}-h\right), \quad M S E_{h}=\frac{1}{N} \sum_{i=1}^{N}\left(\hat{h}_{i}-h\right)^{2} .
$$

The results of this simulation study are given in Figures 3 and 4 .

From these figures, we observe that when $n$ increases, the empirical biases and MSEs approach to 0 in all cases.

\section{Applications}

In this section, we prove empirically the flexibility of the TSCW distribution by considering three real life data sets. In particular, we aim to compare the TSCW model related to the TSCW distribution with the competitive models listed in Table 3. The definitions of the pdfs of these models are given in Appendix. The goodness-of-fit of these model is explored by considering the following measures: $-\widehat{\ell}$ (the maximized log-likelihood), AIC (Akaike information criterion), BIC (Bayesian information criterion), CVM (Cramér-Von Mises), AD (Anderson-Darling) and KS (Kolmogorov Smirnov) statistics with its p-value (PV). The numerical results in this section are obtained by using $\mathrm{R}$ programs.

The first data set contains exact times of failure. More precisely, it consists in a set of the life of fatigue fracture of Kevlar 373/epoxy that are subject to constant 

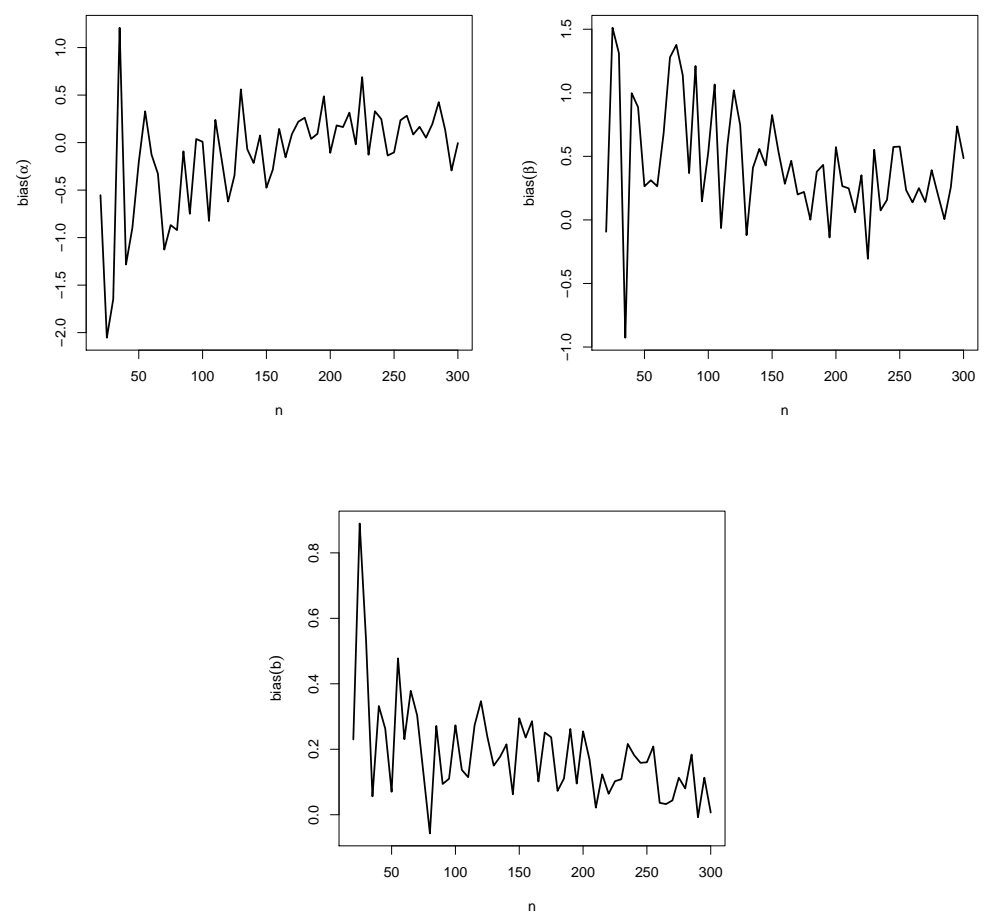

FigurE 3. The biases for the selected parameter values for the TCSW distribution.

TABLE 3. The competitive models of the TSCW distribution

\begin{tabular}{ll}
\hline \hline Distributions & Authors \\
\hline \hline Odd Burr Weibull (OBW) & {$[1]$} \\
CS transformation of exponential $\left(C S 1_{E}\right)$ & {$[7]$} \\
Transmuted Weibull (TW) & {$[4]$} \\
SS transformation of exponential $\left(S S_{E}\right)$ & {$[13]$} \\
\hline \hline
\end{tabular}

pressure (at the $90 \%$ stress level) until all had failed. Analysis of this data set can also be found in [3] and [5]. These data are listed below: 0.0251, 0.0886, 0.0891, $0.2501,0.3113,0.3451,0.4763,0.5650,0.5671,0.6566,0.6748,0.6751,0.6753,0.7696$, $0.8375,0.8391,0.8425,0.8645,0.8851,0.9113,0.9120,0.9836,1.0483,1.0596,1.0773$, $1.1733,1.2570,1.2766,1.2985,1.3211,1.3503,1.3551,1.4595,1.4880,1.5728,1.5733$, $1.7083,1.7263,1.7460,1.7630,1.7746,1.8275,1.8375,1.8503,1.8808,1.8878,1.8881$, $1.9316,1.9558,2.0048,2.0408,2.0903,2.1093,2.1330,2.2100,2.2460,2.2878,2.3203$, $2.3470,2.3513,2.4951,2.5260,2.9911,3.0256,3.2678,3.4045,3.4846,3.7433,3.7455$, 

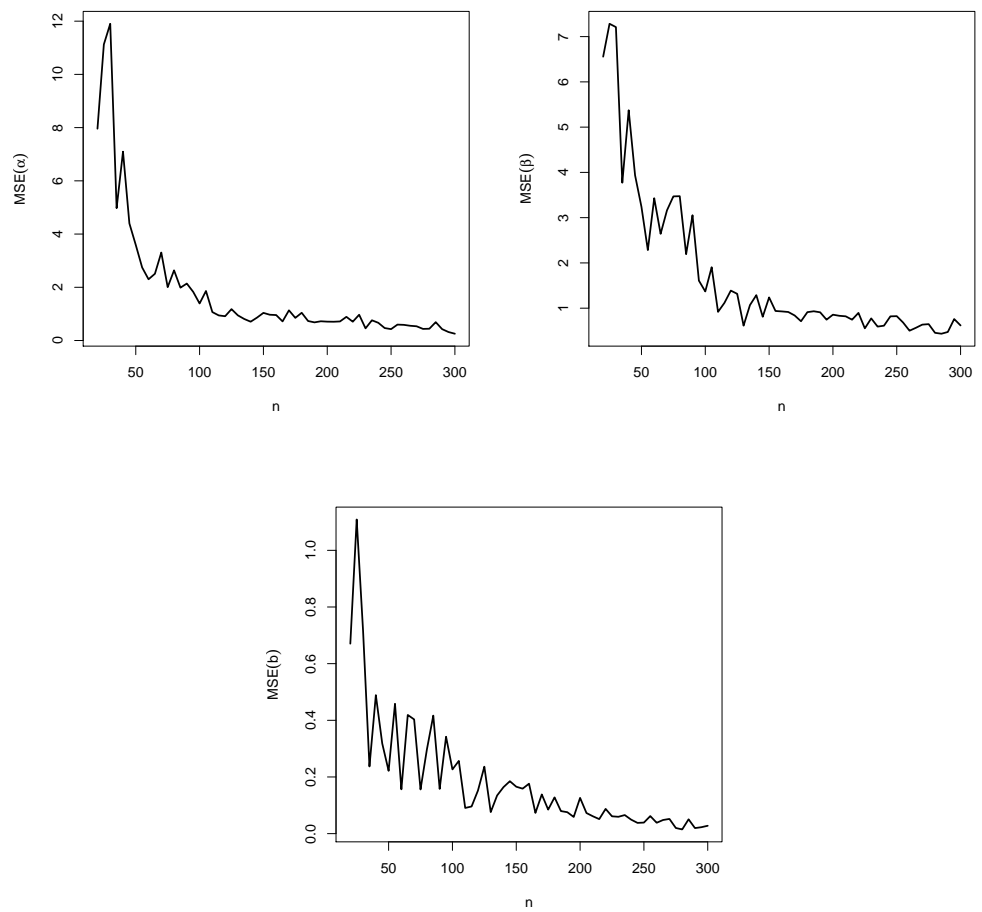

FiguRE 4. The MSEs for the selected parameter values for the TCSW distribution.

$3.9143,4.8073,5.4005,5.4435,5.5295,6.5541,9.0960$.

The second data set represents the total milk production in the first birth of 107 cows from SINDI race. Further details are given in [8]. These data are listed below: 0.4365, 0.4260, 0.5140, 0.6907, 0.7471, 0.2605, 0.6196, 0.8781, 0.4990, 0.6058, 0.6891, $0.5770,0.5394,0.1479,0.2356,0.6012,0.1525,0.5483,0.6927,0.7261,0.3323,0.0671$, $0.2361,0.4800,0.5707,0.7131,0.5853,0.6768,0.5350,0.4151,0.6789,0.4576,0.3259$, $0.2303,0.7687,0.4371,0.3383,0.6114,0.3480,0.4564,0.7804,0.3406,0.4823,0.5912$, $0.5744,0.5481,0.1131,0.7290,0.0168,0.5529,0.4530,0.3891,0.4752,0.3134,0.3175$, 0.1167, 0.6750, 0.5113, 0.5447, 0.4143, 0.5627, 0.5150, 0.0776, 0.3945, 0.4553, 0.4470, $0.5285,0.5232,0.6465,0.0650,0.8492,0.8147,0.3627,0.3906,0.4438,0.4612,0.3188$, $0.2160,0.6707,0.6220,0.5629,0.4675,0.6844,0.3413,0.4332,0.0854,0.3821,0.4694$, $0.3635,0.4111,0.5349,0.3751,0.1546,0.4517,0.2681,0.4049,0.5553,0.5878,0.4741$, $0.3598,0.7629,0.5941,0.6174,0.6860,0.0609,0.6488,0.2747$. 
The third data set is given by [12] and recently [18] use this data, consists of thirty successive values of March precipitation (in inches) in Minneapolis/StPaul. The data are listed below: $0.77,1.74,0.81,1.2,1.95,1.2,0.47,1.43,3.37,2.2,3$, $3.09,1.51,2.1,0.52,1.62,1.31,0.32,0.59,0.81,2.81,1.87,1.18,1.35,4.75,2.48$, $0.96,1.89,0.9,2.05$.

For the data sets 1, 2 and 3 respectively,

- Tables 4, 6 and 8 provide the values of goodness-of-fit measures for the TSCW model and the competitors.

- The MLEs of the parameters, as well as their corresponding standard errors (SEs) (in parentheses), are listed in Tables 5, 7 and 9.

Analysis of these results indicates that the TSCW model yields the best fit to the data sets 1, 2 and 3 compared to the other models. In particular, for each of the data set, it has the lowest AIC, BIC and the greatest PV.

- The plots of the fitted TSCW model are shown in Figures 5, 6 and 7.

TABLE 4. Goodness-of-fit measures for data set 1.

\begin{tabular}{lccccccc}
\hline \hline Model & $-\widehat{\ell}$ & AIC & BIC & CVM & AD & KS & PV \\
\hline TCSW & 120.4692 & 246.9384 & 253.9306 & 0.0694 & 0.4177 & 0.0748 & 0.7597 \\
OBW & 122.5244 & 253.0488 & 262.3717 & 0.1305 & 0.7672 & 0.1106 & 0.2882 \\
$C S 1_{E}$ & 128.8944 & 263.7889 & 270.7811 & 0.3159 & 1.8822 & 0.1261 & 0.1632 \\
TW & 121.7353 & 249.4706 & 256.4628 & 0.1062 & 0.6292 & 0.0957 & 0.4600 \\
$S S_{E}$ & 125.4989 & 252.9978 & 255.3285 & 0.1155 & 0.6836 & 0.1490 & 0.0614 \\
\hline \hline
\end{tabular}


TABLE 5. MLEs and SEs (in parentheses) for data set 1.

\begin{tabular}{lcccc}
\hline \hline Model & \multicolumn{4}{c}{ Estimates } \\
\hline TCSW & 0.1492 & 1.5292 & 0.1304 & \\
$(\alpha, \beta, b)$ & $(0.0288)$ & $(0.1486)$ & $(0.0835)$ & \\
OBW & 10.1144 & 84.8733 & 0.0157 & 0.9720 \\
$(\alpha, \beta, a, b)$ & $(7.6320)$ & $(16.7768)$ & $(0.0216)$ & $(0.0233)$ \\
$C S 1_{E}$ & 0.4739 & 2.0472 & 0.8540 & \\
$(\alpha, \theta, \lambda)$ & $(0.8942)$ & $(7.4751)$ & $(0.1095)$ & \\
TW & 0.4739 & 2.0472 & 0.8540 & \\
$(\eta, \delta, \lambda)$ & $(0.8942)$ & $(7.4751)$ & $(0.1095)$ & \\
$S S_{E}$ & 0.2881 & & & \\
$(\theta)$ & $(0.0309)$ & & & \\
\hline \hline
\end{tabular}

TABLE 6. Goodness-of-fit measures for data set 2.

\begin{tabular}{lccccccc}
\hline \hline Model & $-\widehat{\ell}$ & AIC & BIC & CVM & AD & KS & PV \\
\hline TCSW & -24.4397 & -42.8795 & -34.8689 & 0.1438 & 0.9826 & 0.0603 & 0.8306 \\
OBW & -21.3459 & -34.6919 & -24.0006 & 0.2328 & 1.5297 & 0.0832 & 0.4490 \\
$C S 1_{E}$ & -22.9599 & -39.9197 & -31.9013 & 0.1541 & 1.0697 & 0.0788 & 0.7601 \\
TW & -22.9188 & -22.8377 & -21.8192 & 0.1815 & 1.2233 & 0.0677 & 0.7122 \\
$S S_{E}$ & -24.3728 & -36.7458 & -32.4474 & 0.9567 & 6.1905 & 0.1424 & 0.0005 \\
\hline \hline
\end{tabular}

TABLE 7. MLEs and SEs (in parentheses) for data set 2.

\begin{tabular}{lcccc}
\hline \hline Model & \multicolumn{4}{c}{ Estimates } \\
\hline TCSW & 6.2153 & 2.2506 & 0.8059 & \\
$(\alpha, \beta, b)$ & $(0.7736)$ & $(0.2221)$ & $(0.0640)$ & \\
OBW & 12.2141 & 73.0527 & 0.0813 & 0.8944 \\
$(\alpha, \beta, a, b)$ & $(2.1468)$ & $(6.1504)$ & $(0.0590)$ & $(0.0349)$ \\
$C S 1_{E}$ & 0.2107 & 9.6883 & 0.1109 & \\
$(\alpha, \theta, \lambda)$ & $(4.3198)$ & $(1.9894)$ & $(0.2559)$ & \\
TW & 0.4717 & 2.3229 & -0.4801 & \\
$(\eta, \delta, \lambda)$ & $(0.0290)$ & $(0.2387)$ & $(0.2083)$ & \\
$S S_{E}$ & 2.2553 & & & \\
$(\theta)$ & $(0.1482)$ & & & \\
\hline \hline
\end{tabular}


TABLE 8. Goodness-of-fit measures for data set 3.

\begin{tabular}{lccccccc}
\hline \hline Model & $-\widehat{\ell}$ & AIC & BIC & CVM & AD & KS & PV \\
\hline TCSW & 38.3101 & 82.6201 & 86.8237 & 0.0156 & 0.1212 & 0.0606 & 0.9999 \\
OBW & 38.6427 & 85.2855 & 90.8903 & 0.0219 & 0.1692 & 0.0689 & 0.9988 \\
$C S 1_{E}$ & 41.8522 & 89.7045 & 93.9081 & 0.0702 & 0.4887 & 0.0964 & 0.9429 \\
TW & 38.3987 & 82.7978 & 87.0011 & 0.0172 & 0.1350 & 0.0750 & 0.9978 \\
$S S_{E}$ & 44.3714 & 92.7428 & 95.5452 & 0.0137 & 0.1057 & 0.2203 & 0.1087 \\
\hline \hline
\end{tabular}

TABlE 9. MLEs and SEs (in parentheses) for data set 3.

\begin{tabular}{lcccc}
\hline \hline Model & \multicolumn{4}{c}{ Estimates } \\
\hline TCSW & 0.1574 & 2.1094 & 0.2480 \\
$(\alpha, \beta, b)$ & $(0.0826)$ & $(0.2773)$ & $(0.2434)$ & \\
OBW & 10.2204 & 84.7145 & 0.0215 & 0.9706 \\
$(\alpha, \beta, a, b)$ & $(3.2284)$ & $(9.1948)$ & $(0.0066)$ & $(0.0236)$ \\
$C S 1_{E}$ & 1.4770 & 17.0918 & 0.5383 & \\
$(\alpha, \theta, \lambda)$ & $(4.0750)$ & $(4.7156)$ & $(0.0065)$ & \\
TW & 2.3166 & 1.9458 & 0.6174 & \\
$(\eta, \delta, \lambda)$ & $(0.5886)$ & $(0.2698)$ & $(0.6530)$ & \\
$S S_{E}$ & 3.6441 & & & \\
$(\theta)$ & $(0.1447)$ & & \\
\hline \hline
\end{tabular}




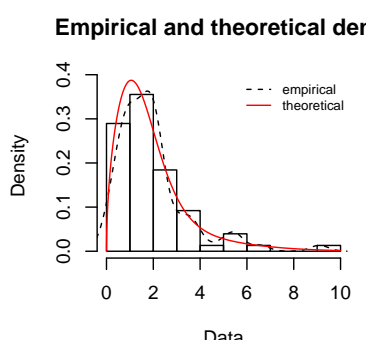

Empirical and theoretical CDFs

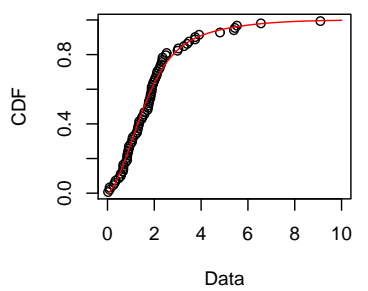

Q-Q plot

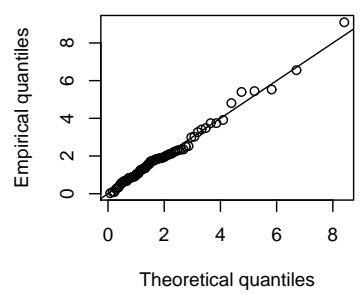

P-P plot

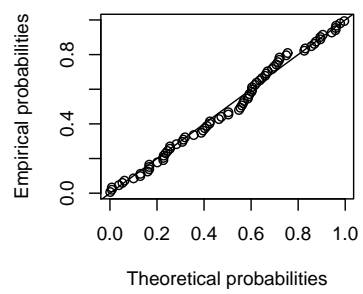

Figure 5. PP, QQ, epdf and ecdf plots of the TSCW distribution for data set 1.

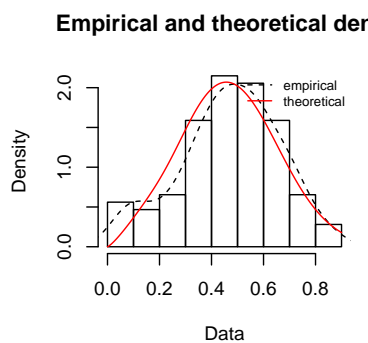

Empirical and theoretical CDFs

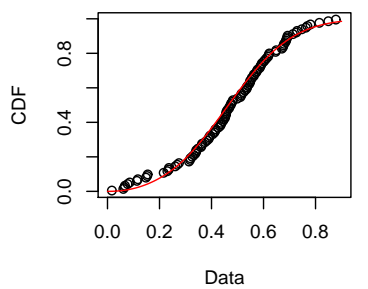

Q-Q plot

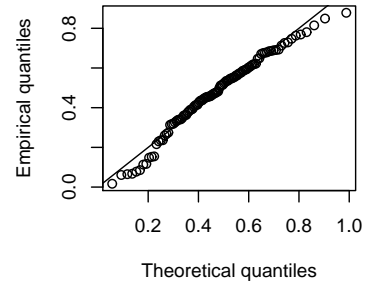

P-P plot

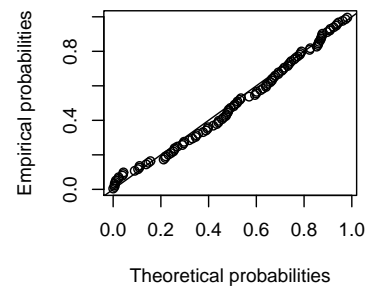

Figure 6. PP, QQ, epdf and ecdf plots of the TSCW distribution for data set 2 . 

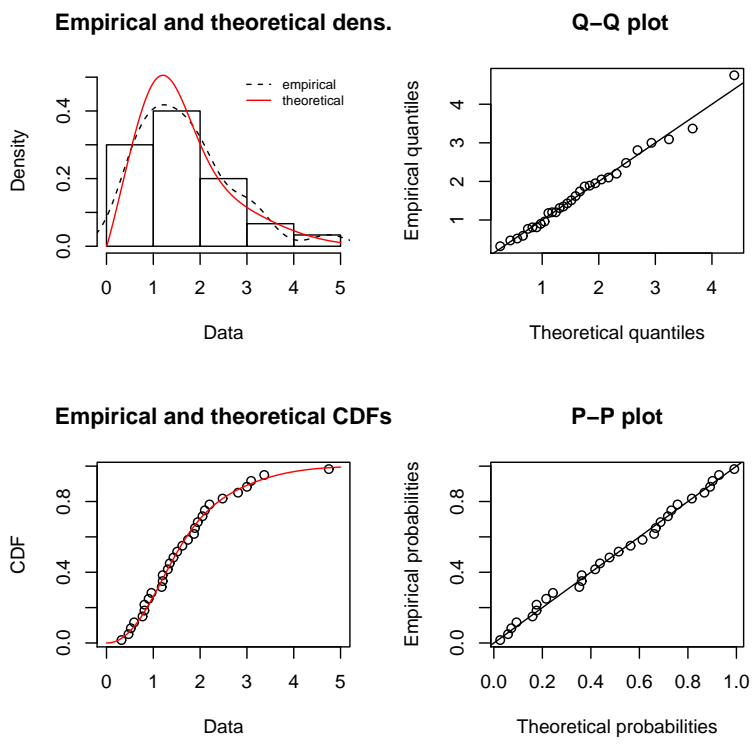

Figure 7. PP, QQ, epdf and ecdf plots of the TSCW distribution for data set 3 . 


\section{Proofs}

Proof of Theorem 2.1. It is clear that $B(0)=0, B\left(\frac{\pi}{2}\right)=1$ and, for any $y \in\left[0, \frac{\pi}{2}\right]$, $\cos (y)+\left(c+y e^{-a y}\right) \sin (y)>0$. Let us now prove that $B(y)$ is a nondecreasing function for $y \in \mathbb{R}$. Let us observe that $B(y)$ is differentiable for $y \in \mathbb{R}$ excepted, eventually, at the points 0 and $\frac{\pi}{2}$ (depending on the values of the parameters $a, b$ and $c$ ). Owing to the quotient rule for derivatives, for any $y \in\left(0, \frac{\pi}{2}\right)$, we obtain

$$
B^{\prime}(y)=A^{\prime}(y)=\left(c+\frac{\pi}{2} e^{-a \frac{\pi}{2}}\right) \frac{A_{1}(y)}{A_{2}(y)},
$$

where

$$
\begin{aligned}
A_{1}(y) & =\left(\sin (y)-b y e^{-a y} \cos (y)\right)^{\prime}\left(\cos (y)+\left(c+y e^{-a y}\right) \sin (y)\right) \\
& -\left(\sin (y)-b y e^{-a y} \cos (y)\right)\left(\cos (y)+\left(c+y e^{-a y}\right) \sin (y)\right)^{\prime}
\end{aligned}
$$

and $A_{2}(y)=\left(\cos (y)+\left(c+y e^{-a y}\right) \sin (y)\right)^{2}$.

We have $A_{2}(y)>0$. Let us investigate the sign of $A_{1}(y)$. By an expansion of their terms and the trigonometric identities: $2 \sin (y) \cos (y)=\sin (2 y), \cos ^{2}(y)=\frac{1+\cos (2 y)}{2}$ and $\sin ^{2}(y)=\frac{1-\cos (2 y)}{2}$, we obtain

$$
\begin{aligned}
& A_{1}(y)= \\
& \left(\cos (y)-b e^{-a y} \cos (y)+a b e^{-a y} y \cos (y)+b e^{-a y} y \sin (y)\right)\left(\cos (y)+\left(c+y e^{-a y}\right) \sin (y)\right) \\
& -\left(\sin (y)-b y e^{-a y} \cos (y)\right)\left(\left(c+e^{-a y} y\right) \cos (y)-\sin (y)+\left(e^{-a y}-a e^{-a y} y\right) \sin (y)\right) \\
& =\frac{1}{2} e^{-2 a y}\left(2 e^{2 a y}+2 b y^{2}+e^{a y}(a y-1+b(a y-1+2 c y)+(a y-1)((b-1) \cos (2 y)+b c \sin (2 y)))\right. \\
& =\frac{1}{2} e^{-2 a y}\left(2 e^{2 a y}+2 b y^{2}+2 b c y e^{a y}-e^{a y}(1-a y)(1+b-(1-b) \cos (2 y)+b c \sin (2 y))\right) .
\end{aligned}
$$

Since $b \in[0,1], c \geq 0$ and $y \in\left(0, \frac{\pi}{2}\right)$, we have $1-(1-b) \cos (2 y) \geq 0$ and $b c \sin (2 y) \geq 0$. Let us now distinguish the case $y \geq \frac{1}{a}$ and the case $y<\frac{1}{a}$.

If $y \geq \frac{1}{a}$, we have $e^{a y}(1-a y) \leq 0$. Therefore, $A_{1}(y) \geq 0$ as a sum of positive functions.

If $y<\frac{1}{a}$, we have $e^{a y}(1-a y)>0$ and, by the inequality $e^{x} \geq 1+x, x \in \mathbb{R}$, with $x=-a y$, we get $e^{a y}(1-a y) \leq 1$. On the other side, we have $-(1-b) \cos (2 y) \leq 1-b$ and, the inequality $\sin (x) \leq x, x \geq 0$, gives $b c \sin (2 y) \leq 2 b c y$. Therefore,

$$
\begin{aligned}
A_{1}(y) & \geq \frac{1}{2} e^{-2 a y}\left(2 e^{2 a y}+2 b y^{2}+2 b c y e^{a y}-(1+b+(1-b)+2 b c y)\right) \\
& =e^{-2 a y}\left(\left(e^{2 a y}-1\right)+b y^{2}+b c y\left(e^{a y}-1\right)\right) .
\end{aligned}
$$

Since $a \geq 0$ and $y \geq 0$, we have $e^{2 a y} \geq 1$ and $e^{a y} \geq 1$. Hence, $A_{1}(y) \geq 0$ as a sum of positive functions. This proves $A^{\prime}(y) \geq 0$ for any $y \in\left(0, \frac{\pi}{2}\right)$, implying that $B^{\prime}(y) \geq 0$, so $B(y)$ is a nondecreasing function for $y \in \mathbb{R}$. This ends the proof of Theorem 2.1. 


\section{APPENDIX}

The considered pdf of Section 4 are given below.

- Odd Burr Weibull (OBW) by [1]:

$g(x)=\alpha \beta a b x^{\alpha-1}\left(1+x^{\alpha}\right)^{\beta b-1}\left[1-\left(1+x^{\alpha}\right)^{-\beta}\right]^{b-1} e^{-a\left[\left(1+x^{\alpha}\right)^{\beta}-1\right]^{b}}, \quad \alpha, \beta, a, b, x>0$.

- CS transformation of exponential $\left(C S 1_{E}\right)$ by [7]:

$$
g(x)=\frac{2 \pi \alpha\left(\alpha \sin \left(\frac{\pi}{2} e^{-\frac{x}{\lambda}}\right)+\theta\left(\cos \left(\frac{\pi}{2} e^{-\frac{x}{\lambda}}\right)\right)^{3}\right) e^{-\frac{x}{\lambda}}}{\lambda\left(2 \alpha+\theta \sin \left(\pi e^{-\frac{x}{\lambda}}\right)\right)^{2}}, \quad \alpha, \theta, \lambda, x>0 .
$$

- Transmuted Weibull (TW) by [4]:

$$
g(x)=\frac{\eta}{\delta}\left(\frac{x}{\delta}\right)^{\eta-1} e^{-\left(\frac{x}{\delta}\right)^{\eta}}\left[1-\lambda+2 \lambda e^{-\left(\frac{x}{\delta}\right)^{\eta}}\right], \quad \eta, \delta, \lambda, x>0 .
$$

- SS transformation of exponential $\left(S S_{E}\right)$ by [13]:

$$
g(x)=\frac{\pi}{2} \theta e^{-\theta x} \sin \left(\frac{\pi}{2} e^{-\theta x}\right), \quad \theta, x>0 .
$$

\section{REFERENCES}

[1] A.Z. Afify, G.M. Cordeiro, E.M.M. Ortega, H.M. Yousof and N.S. Butt, The four-parameter Burr XII distribution: Properties, regression model and applications, Communications in Statistics Theory and Methods, 47(2018), No. 11, 2605-2624.

[2] R.Q. Al-Faris and S. Khan, Sine square distribution: A new statistical model based on the sine function, Journal of Applied Probability and Statistics, 3(2008), No. 1, 163-73.

[3] D.F. Andrews and A.M. Herzberg, Data: A Collection of Problems from Many Fields for the Student and Research Worker, Springer Series in Statistics, New York, (1985).

[4] G. Aryal and C.P. Tsokos, Transmuted Weibull Distribution: A Generalization of the Weibull Probability Distribution, European journal of pure and applied mathematics, 4(2011), No. 2, 89-102.

[5] R.E. Barlow, R.H. Toland and T. Freeman, A Bayesian analysis of stressrupture life of kevlar 49/epoxy spherical pressure vessels, in 'Proc. Conference on Applications of Statistics', Marcel Dekker, New York, (1984).

[6] S. Chakraborty, P.J. Hazarika and M.M. Ali, A new skew-logistic distribution and its properties, Pakistan Journal of Statistics, 28(2012), No. 4, 513-524.

[7] C. Chesneau, H.S. Bakouch and T. Hussain, A new class of probability distributions via cosine and sine functions with applications, Communications in Statistics - Simulation and Computation, (2018), to appear. DOI: 10.1080/03610918.2018.1440303.

[8] G.M. Cordeiro and R.D.S. Brito, The beta power distributions, Brazilian journal of probability and statistics, 26(2012), No. 1, 88-112.

[9] M. Evans, N. Hastings and B. Peacock, Statistical Distributions, 3rd ed. New York: Wiley, (2000), 189-191.

[10] Y.M. Gómez, H. Bolfarine and H.W. Gómez, A New Extension of the Exponential Distribution, Revista Colombiana de Estadstica, 37(2014), No. 1, 25-34. 
[11] G.K. Gilbert, The moon's face; a study of the origin of its features, Bulletin of the Philosophical Society of Washington, Washington, 12(1895), 241-292.

[12] D. Hinkley, On quick choice of power transformation, Applied Statistics, 26(1977), 67-69.

[13] D. Kumar, U. Singh and S.K. Singh, A new distribution using sine function - its application to bladder cancer patients data, J. Stat. Appl. Pro., 4(2015), No. 3, 417-427.

[14] S. Nadarajah and S. Kotz, Beta trigonometric distributions, PEZ, 5(2006), 207-224.

[15] D.H. Raab and E.H. Green, A cosine approximation to the normal distribution, Psychometrika, 26(1961), No. 4, 447-450.

[16] M. Rasekhi, M. Alizadeh, E. Altun, G.G. Hamedani, A.Z. Afify and M. Ahmad, The Modified Exponential Distribution with Applications, Pakistan Journal of Statistics, 33(2017), No. 5, 383-398.

[17] T. Strukov, Shift estimation and forms of Von Mises distribution, Journal of Mathematical Sciences, 189(2013), No. 6, 992-996.

[18] A. Yusuf, B.B. Mikail, A.I. Aliyu and A.L. Sulaiman, The Inverse Burr Negative Binomial Distribution with Application to Real Data, Statistics: A Journal of Theoretical and Applied Statistics, 5(2016), No. 1, 53-65.

${ }^{1}$ Department of Statistics, Govt. S.A Postgraduate College Dera Nawab Sahib, BahaWalpur, Punjab 63360, PAKISTAn, E-MAIl: farrukhjamalmphil@gmail.com

${ }^{2}$ Lmno, University of Caen Normandie, 14032, Caen, France 